\title{
Secure Chained Threshold Proxy Signature without and with Supervision
}

\author{
Zoe L. JIANG, S. M. YIU, Y. DONG, L. C. K. HUI, S. H. Y. WONG
}

Department of Computer Science, The University of Hong Kong, Hong Kong, China. Email: \{ljiang, smyiu, ydong, hui, shywong\}@es.hku.hk.

Received June $4^{\text {th }}, 2009$; revised July $15^{\text {th }}, 2009$; accepted July $24^{\text {th }}, 2009$.

\begin{abstract}
Threshold Proxy Signature (TPS) scheme facilitates a manager to delegate his signing capability to a group of $n_{2}$ subordinates without revealing his own private key, such that a subgroup of at least $t_{2} \leqslant n_{2}$ subordinates is required to generate a proxy signature. In reality, the situation can be more complicated. First of all, the subgroup may further delegate their proxy signing capabilities to another group of $n_{3}$ subordinates such that at least another subgroup of at least $t_{3} \leq n_{3}$ subordinates are of the proxy signing capabilities (in the form of a chain). $t_{2}$ can be unequal to $t_{3}$ depending on the concrete requirement. This is a group-to-group delegation problem. In addition, a supervising agent (SA) may be introduced in the above chain to supervise the subordinates, such that proxy signing can only be successfully executed with SA's agreement. This is a delegation with supervision problem in the threshold delegation chain described above. These two extensions of delegation problems are not solved yet. This paper designs two provably secure cryptographic schemes Chained Threshold Proxy Signature (CTPS) scheme and Chained Threshold Proxy Signature with Supervision $(C T P S w S)$ scheme to solve these two delegation problems.
\end{abstract}

Keywords: Delegation, Threshold Proxy Signature, Chained Threshold Proxy Signature with Supervision

\section{Introduction}

\subsection{Motivation}

It is a common practice for a manager to delegate his signing right to a group of $n$ subordinates when he is on leave so that a subgroup of at least $t \leq n$ of them can cooperate to sign a document on behalf of the manager. This is a threshold delegation problem, and can be solved by Threshold Proxy Signature (TPS) [2] scheme. In reality, the delegation may involve more than one level (in the form of a chain). Consider the following scenario. There is an email sent by the manager of software development department in corporation A, Simon, to the manager of the same department in corporation B, Sam. Since Sam is too busy to check every single detail of the data part before he replies with his signature, and the data are so important that he cannot rely on any single one of his three vice managers (his subordinates), he forwards this email to all of them. Further any two of them, as a subgroup, may forward this email to their employees checking the data part. As a result, to answer this email to Simon, it is desirable for a subgroup of the employees to cooperate on behalf of Sam. How the any two of the

${ }^{*}$ The preliminary work has been published in 2008 International Conference on Computer Science and Software Engineering [1]. vice managers pass their proxy signing capabilities to their employees is referred to group-to-group delegation problem. In a more cautious situation, the contract part needs to be authorized by the manager of the software maintenance department in corporation B, Steven. In this case, besides delegation, Sam is also required to appoint Steven as his supervising agent (SA) such that only when Steven agrees to the contract part, the employees can compute a proxy signature on behalf of Sam. How Sam appoints Steven as an SA is referred to delegation with supervision problem in threshold delegation chain. In this paper, we propose two schemes, Chained Threshold Proxy Signature (CTPS) scheme and Chained Threshold Proxy Signature with Supervision (CTPSwS) scheme, to solve these two delegation problems.

\subsection{Related work}

Mambo et al. [3] introduced the first efficient proxy signature in 1996, where it allows a user to delegate his signing power to a designated signer, a proxy signer. It is widely applicable in all kinds of known standard signature schemes such as El Gamal scheme [4], Okamoto scheme [5] and Fiat-Shamir scheme [6]. In 1997, Kim et al. [2] proposed proxy signature for partial delegation with warrant combining the benefit of Mambo's partial delegation and Neuman's [7] delegation by warrant/certi- 
ficate. They also extended it to a $(t, n)$ Threshold Proxy Signature (TPS) such that any $t \leq n$ proxy signers using their proxy secret key shares can cooperate to generate a proxy signature on behalf of an original signer, but less than $t$ can not, by deploying Ceredo's Schnorr type threshold digital signature scheme [8].

Lui et al. [9] proposed a chained delegation scheme with supervision scheme, in which the original signer sends, in ahead of time, his permission information about the proxy signer to a supervising agent (SA) who he trusts. Then the proxy signer can only generate a valid proxy signature under SA's supervision even when the original signer is not available. This delegation can be executed in multiple levels. However, on one hand, the scheme does not consider the threshold problem. On the other hand, the scheme sacrificed both the original signer and the supervising agent's undeniabilities due to the advantage the authors presented that there is no need for the verifier to be aware of whether supervision is performed or not. In many cases, this is unacceptable from the security point of view.

Boldyreva [10] defined a formal proof model for the security of proxy signature schemes, which enables the cryptographic analysis of such schemes, instead of just presenting attacks that fail. Then they proved the security of Triple Schnorr Proxy Signature scheme, a variant of Kim at al.'s proxy signature scheme, preserving its efficiency, in the random oracle model assuming the hardness of computation of discrete logarithm.

\subsection{Our Contribution}

Firstly, in this paper we propose Chained Threshold Proxy Signature (CTPS) scheme to solve the group-to-group delegation problem. Although, Threshold Proxy Signature (TPS) scheme [2] and Triple Schnorr Signature scheme [10] are two important components to design our scheme, we need to consider how to distribute the proxy shares from a subgroup of vice managers to another subgroup of employees in a group-to-group manner. Therefore, we deploy Herzberg et al.'s [11] proactive secret sharing idea into our scheme. Proactive secret sharing is proposed to periodically renew the shares without changing the secret, in such a way that any information learnt by the adversary about individual shares becomes obsolete after the shares are renewed. But in our scheme, the renewed shares should be securely passed to employees by each vice manager while old ones are kept secret by the vice managers themselves.

To solve the delegation with supervision problem in threshold delegation chain, we adapt Lui et al's supervision idea in delegation chain (no threshold) into CTPS scheme to implement Chained Threshold Proxy Signature with Supervision (CTPSwS) scheme. Different with Lui et al's idea, however, supervising agent is also actively involved in the delegation using his/her own pri- vate key, such that verification for the proxy signature requires supervising agent's public key as well, besides original signer and proxy signers' public keys.

We also provide formal security models and proofs to show that the schemes we designed are secure in the random oracle model assuming the hard problem of discrete logarithm.

\subsection{Organization}

A 3-level CTPS scheme and its security proof will be described in section $2 \& 3$, respectively. Then a 3-level CTPSwS scheme and security proof draft will be introduced in section $4 \& 5$, respectively. Section 6 concludes the paper and discusses some future work.

\section{Chained Threshold Proxy Signature (CTPS)}

Recall that Sam, the manager of software development department in corporation $\mathrm{B}$, delegates his signing right to a group of $n_{2}$ vice managers, any subgroup of whom with $t_{2}$ members further delegate their proxy signing capabilities to a group of $n_{3}$ employees, such that any subgroup of $t_{3}$ employees can reply to Simon with their proxy signature on behalf of Sam.

We can formally define the above roles by letting Sam the original signer $u_{1}$ in level 1 , vice managers a group of $n_{2}$ proxy signers in level $2,\left(\left\{u_{2, j}\right\}_{n_{2}}\right.$ for short $)$, and employees a group of $n_{3}$ proxy signers in level 3, ( $\left\{u_{3, k}\right\}_{n_{3}}$ for short). Any subgroup of $t_{2} \leq n_{2}$ vice managers performing the delegation is defined as $U_{2}$. Similarly, any subgroup of $t_{3} \leq n_{3}$ employees signing the replied email is defined as $U_{3}$. Note the difference between $\left\{u_{2, j}\right\}_{n_{2}}$ and $U_{2}, \quad\left\{u_{3, k}\right\}_{n_{3}}$ and $U_{3}$. WLOG, we assume $U_{2}=\left\{u_{2,1}, u_{2,2}, \cdots, u_{2, t_{2}}\right\}=\left\{u_{2, j}\right\}_{t_{2}}$ and $U_{3}=\left\{u_{3,1}, u_{3,2}, \cdots, u_{3, t_{3}}\right\}$ $=\left\{u_{3, k}\right\}_{t_{3}}$. Let $\left(s k_{1}, p k_{1}\right),\left(s k_{2, j}, p k_{2, j}\right),\left(s k g_{2}, p k g_{2}\right),\left(s k_{3, k}\right.$, $\left.p k_{3, k}\right)$ and $\left(\operatorname{skg}_{3}, p k g_{3}\right)$ denote $u_{1}, u_{2, j},\left\{u_{2, j}\right\}_{n_{2}}, u_{3, k}$, and $\left\{u_{3, k}\right\}_{n_{3}}$ 's secret/public key pairs respectively. By extending Boldyreva's proxy signature scheme model, CTPS scheme to achieve the delegation procedure should involve a one-to-group protocol run between the original signer and the group of proxy signers in level 2, a group-to-group protocol run between any subgroup of proxy signers in level 2 and the group of proxy signers in level 3, a chained threshold proxy signing algorithm and the corresponding verification algorithm. Additionally, there should be an algorithm that extracts the identities of the groups of proxy signers in both level $2 \& 3$.

Definition 1 describes the detailed components of a 3-level Chained Threshold Proxy Signature scheme. A list of important parameters and symbols is shown here for your reference.

$\omega_{1}$ : warrant including $u_{1}$ and $\left\{u_{2, j}\right\}_{n_{2}}$ 's IDs, and other 
information on the delegation

$\omega_{2}$ : warrant including $\left\{u_{2, j}\right\}_{n_{2}}$ and $\left\{u_{3, k}\right\}_{n_{3}}$ 's IDs, and

other information on the delegation

$s k t$ : secret key transformation generated by $u_{1}$

$s k t_{1, j}$ : share of secret key transformation sent to $u_{2, j}$

$s k p_{2, j}$ : proxy secret key share generated by $u_{2, j}$

$s k t_{2, j}$ : share of proxy secret key transformation generated by $u_{2, j}$

$s k t_{2, j, k}$ : sub-share of proxy secret key transformation sent to $u_{3, k}$

$s k t_{2, k}^{\prime}:$ share of proxy secret key transformation retrieved by $u_{3, k}$

$s k p_{3, k}$ : proxy secret key share generated by $u_{3, k}$

$p \sigma_{3}$ : chained threshold proxy signature.

Definition 1 (CTPS Scheme) Let CTPS $=(G, K,(T D$, $T P)$, (CTD, CTP), CTPS, CTPV, CTPID) be a chained threshold proxy signature scheme, where the constituent algorithms run in polynomial time.

$\underline{G}$ is a random parameter-generation algorithm, and it will output some global parameters params.

$\underline{K}$ is a random key-generation algorithm, and it will output secret/public key pairs for original signer $u_{1}$ and proxy signers in both level $2 \& 3,\left\{u_{2, j}\right\}_{n_{2}}$ and $\left\{u_{3, k}\right\}_{n_{3}}$, in the scheme.

$(T D, T P)$ is a Threshold Designation-Proxy protocol between the original signer $u_{1}$ and the proxy signers in level 2, $\left\{u_{2, j}\right\}_{n_{2}}$. Both $T D$ and $T P$ take as input the public keys $p k_{1}$ and $p k g_{2}$, respectively. $T D$ also takes as input the secret key $s k_{1}$ of $u_{1}$, and $T P$ also takes as input the secret key $s k_{2, j}$ of $u_{2, j}$. As the result of the interaction, the expected local output of $T P$ is $s k p_{2, j}$, the proxy secret share which is kept secret by each $u_{2, j}$.

$$
\left[T D\left(p k_{1}, p k g_{2}, s k_{1}\right), T P\left(p k_{1}, p k g_{2}, s k_{2, j}\right)\right] \rightarrow s k p_{2, j}
$$

$(C T D, C T P)$ is a Chained Threshold DesignationProxy protocol between $U_{2}$ and $\left\{u_{3, k}\right\}_{n_{3}}$. Both CTD and CTP take as input the public keys $p k_{1}, \mathrm{pkg}_{2}$ and $\mathrm{pkg}_{3}$, respectively. CTD also takes as input the proxy secret shares $\left\{s k p_{2, j}\right\}_{t_{2}}$ of $\left\{u_{2, j}\right\}_{t_{2}}$. CTP takes as input the secret key $s k_{3, k}$ of $u_{3, k}$. The expected local output of CTP is $s k p_{3, k}$, the proxy secret key share which is kept secret by each $u_{3, k}$. Note that for each $u_{3, k}$ to generate $s k p_{3, k}$, the subgroup $U_{2}$ is involved in $C T D$, but not just a certain proxy signer in $U_{2}$.

$$
\begin{gathered}
{\left[C T D\left(p k_{1}, p k g_{2}, p k g_{3},\left\{s k p_{2, j}\right\}_{t_{2}}\right),\right.} \\
\left.C T P\left(p k_{1}, p k g_{2}, p k g_{3}, s k_{3, k}\right)\right] \rightarrow \operatorname{skp}_{3, k}
\end{gathered}
$$

CTPS is the (possibly) randomized Chained Threshold Proxy Signing algorithm. It takes as input $\left\{s k p_{3, k}\right\}_{t_{3}}$ and a message $M \in\{0,1\}^{*}$, and outputs a chained threshold-proxy signature $p \sigma_{3}$.

$$
\operatorname{CTPS}\left(M,\left\{s k p_{3, k}\right\}_{t_{3}}\right) \rightarrow p \sigma_{3}
$$

CTPV is the deterministic Chained Threshold Proxy Verification algorithm. It takes as input a message $M$, a proxy signature $p \sigma_{3}$, and $\left(p k_{1}, p k g_{2}, p k g_{3}\right)$, and outputs 0 or 1.

$$
\operatorname{CTPV}\left(M, p \sigma_{3}, p k_{1}, p k g_{2}, p k g_{3}\right)=0 / 1
$$

CTPID is the Chained Threshold Proxy IDentification algorithm. It takes as input a valid proxy signature $p \sigma_{3}$ and outputs identities of two proxy signer groups, i.e., public keys.

$$
\operatorname{CTPID}\left(p \sigma_{3}\right)=\left(p k g_{2}, p k g_{3}\right) / \perp
$$

SIGNATURE VERIFICATION CONDITION: If $C T P V=1$ and $C T P I D=\left(p k g_{2}, p k g_{3}\right)$, we say $p \sigma_{3}$ is a valid chained threshold proxy signature by proxy signers in $U_{2}$ and $U_{3}$ on behalf of $u_{1}$.

The definition clearly describes what kinds of individual algorithms and interactive protocols are required to be run by original signer and proxy signers. After define the structure of CTPS scheme, we design a concrete scheme based on the definition.

We give a high-level description of our scheme here, followed by the concrete calculation. First of all, by using public parameters and secret/public key pairs generated through $G$ and $K, u_{1}$ generates the certificate of warrant $\omega_{1}$ in (1), which is actually a signature of $\omega_{1}$ using $s k_{1}$. We call it secret key transformation $s k t_{1}$ in our scheme since it masks $u_{1}$ 's secret key $s k_{1}$ and will be used for $\left\{u_{2, j}\right\}_{n_{2}}$ to generate proxy signing keys. In order to designate $\left\{u_{2, j}\right\}_{n_{2}}$ as $u_{1}$ 's threshold proxy signers, each share $s k t_{1, j}$ generated by (2) will be distributed to $u_{2, j}$ securely. After verifying $s k t_{1, j}$ as a signature generated by $u_{1}$ using (3), each $u_{2, j}$ computes proxy secret key share $s k p_{2, j}$ in (4).

As the applications we described above, if any $t_{2} \leq n_{2}$ vice managers, such as $U_{2}$, want to further delegate their proxy signing capabilities to their employees $\left\{u_{3, k}\right\}_{n_{3}}$, which we call a group-to-group delegation, each $u_{2, j} \in U_{2}$ computes secret key transformation $s k t_{2, j}$ in (5) as $u_{1}$ does in (1), then divides it to $n_{3}$ shares, $s k t_{2, j, k}\left(k=1,2, \cdots, n_{3}\right)$, as calculated in (6), which are sent to $u_{3, k}$ securely. As a result, each $u_{3, k}$ verifies $s k t_{2, j, k}\left(k=1,2, \cdots, t_{2}\right)$ he receives using (8) and computes $s k t_{2, k}^{\prime}$ by accumulating them in (9). By comparing (5) and (9), $s k t_{2, j}$ and $s k t_{2, k}^{\prime}$ are generated by two different random polynomials $F_{2}(j)$ and $F_{2}^{\prime}(k)$ with same constant $\operatorname{sktg}_{2}$. For how (9) is deduced, please refer to Lagrange Formula, which was also used in [2]. Then each $u_{3, k}$ can successfully generate the proxy secret key share $s k p_{3, k}$ in (11). 
Let us discuss a little more about the difference and difficulty of $(C T D, C T P)$ protocol compared with $(T D$, $T P$ ) here. During $(T D, T P)$ protocol, $s k t_{1, j}$ carrying secret information $s k_{1}$ inside is generated and delivered to $u_{2, j}$ as the mark for $u_{1}$ to designate $u_{2, j}$ as one of his proxy signers. Similarly in $(C T D, C T P)$ protocol, $s k t_{2, j}$ carrying secret information $s k_{2, j}$ should also be delivered to $\left\{u_{3, k}\right\}_{n_{3}}$, but in an indirect way for the reason that there are a group of delegators and a group of delegatees. Sending $s k t_{2, j}$ to $u_{3, k}$ where $j=k$ one by one does not work because $U_{2}$ and $\left\{u_{3, k}\right\}_{n_{3}}$ may have different numbers $t_{2}$ and $n_{2}$.

However, from the group point of view, we need a scheme to reshuffle $\left\{s k t_{2, j}=F_{2}(j)\left(j=1,2, \cdots, t_{2}\right)\right\}$ to $\left\{s k t_{2, k}^{\prime}=F_{2}^{\prime}(k)\left(k=1,2, \cdots, n_{3}\right)\right\}$, satisfying that $F_{2}(0)=F_{2}^{\prime}(0)=s k t g_{2}$. It seems that we keep the group secret key transformation $\mathrm{sktg}_{2}$ unchanged and make secret key transformation shares updated. With this purpose, we found a good candidate of proactive secret sharing approach [11], which was proposed to periodically renew the shares, like $\left\{s k t_{2, j}\right\}_{t_{2}}$, to the new ones, like $\left\{s k t_{2, k}^{\prime}\right\}_{n_{3}}$, without changing the secret, like $s k t g_{2}$. However, in our scheme, the renewed ones belong to $\left\{u_{3, k}\right\}_{n_{3}}$, but not $\left\{u_{2, j}\right\}_{n_{2}}$.

Schnorr signature scheme [12] is used in CTPS and $C T P V$ where partial proxy signatures $\left\{p s_{3, k}\right\}_{t_{3}}$ are published among $U_{3}$ such that each $u_{3, k} \in U_{3}$ can calculate proxy signature $p \sigma_{3}$ in (13). The following details how the algorithms and protocols are performed.

The system runs $G$. On input $1^{k}$, params $=G\left(1^{k}\right)=$ $(p, q, g, G, H)$, such that $2^{k-1} \leq p<2^{k}, q \mid p-1, g \in Z_{p}^{*}$ of order $q$, two hash functions $G:\{0,1\}^{*} \rightarrow Z_{q}$, and $H:\{0,1\}$ * $\rightarrow Z_{q}$.

The system runs $K$. On input $(p, q, g, G, H), K$ generates $s k_{1} \in{ }_{R} Z_{q}, p k_{1}=g^{s k_{1}} \bmod p, s k_{2, j}=S K_{2}(j)$ and $p k_{2, j}=$ $g^{s k_{2, j}} \bmod p$, where $S K_{2}(x)$ is a random polynomial with random constant $s k g_{2}$ and degree $t_{2}-1 . p k g_{2}=g^{s k g_{2}}$ $\bmod p$. Note that although the intuitive idea for the above procedure is to generate $S K_{2}(x)$ and distribute $s k_{2, j}$ to $u_{2, j}$ securely by a trusted dealer, we deploy the protocol for generating random number in [2] to implement it without a dealer for security consideration. As a result, each $u_{2, j}$ can only calculate his own secret key $s k_{2, j}$ without knowing $s k g_{2} . s k g_{3}, p k g_{3},\left\{s k_{3, k}\right\}_{n_{3}}$ and $\left\{p k_{3, k}\right\}_{n_{3}}$ are generated in the same way.

$\underline{u}_{1}$ runs $T D$.

$$
\begin{array}{r}
s k t_{1}=e_{1} \cdot s k_{1}+k_{1} \bmod q, \text { where } \\
r_{1}=g^{k_{1}} \bmod p, k_{1} \in{ }_{R} Z_{q}^{*},
\end{array}
$$

$$
\begin{gathered}
e_{1}=G\left(0\left\|p k_{1}\right\| p k g_{2} \| \omega_{1}, r_{1}\right) . \\
s k t_{1, j}=F_{1}(j)=s k t_{1}+a_{1, j} j+a_{1,2} j^{2}+\cdots+a_{1, t_{2}-1} j^{t_{2}-1} \bmod q .
\end{gathered}
$$

$F_{1}(j)$ is a random polynomial privately owned by $u_{1} . r_{1}$ and $\left\{g^{a_{1, m}} \bmod p\right\} t_{2}-1$ are broadcast.

$\underline{u_{2, j}}$ runs $T P$.

$$
\begin{array}{r}
g^{s k t_{1, j}}=\left(p k_{1}^{e_{1}} r_{1}\right) \cdot A \bmod p, \text { where } \\
A=\prod_{m=1}^{t_{2}-1}\left(g^{a_{1, m}}\right)^{j^{m}} \bmod p . \\
s k p_{2, j}=e_{1} \cdot s k_{2, j}+s k t_{1, j} \bmod q .
\end{array}
$$

$\underline{u_{2, j}}$ runs $C T D$.

$$
\begin{aligned}
& \begin{aligned}
s k t_{2, j} & =e_{2} \cdot s k p_{2, j}+k_{2, j} \bmod q \\
& =e_{2}\left(e_{1} \cdot S K_{2}(j)+F_{1}(j)\right)+K_{2}(j) \\
& =F_{2}(j), \text { where }
\end{aligned} \\
& e_{2}=G\left(0\left\|p k g_{2}\right\| p k g_{3} \| \omega_{2}, r_{2}\right), \\
& F_{2}(0)=e_{2}\left(e_{1} \cdot s k g_{2}+s k t_{1}\right)+k_{2}=s k t g_{2} . \\
& s k t_{2, j, k}=F_{2, j}(k) \bmod q, \text { where }
\end{aligned}
$$

$$
F_{2, j}(k)=s k t_{2, j}+b_{2, j, 1} k+b_{2, j, 2} k^{2}+\cdots+b_{2, j, t_{3}}-1 k^{t_{3}-1} \bmod q . \text { (7) }
$$

$\left\{k_{2, j}\right\}_{n_{2}}$ are generated by running the protocol for generating random number in [2] such that each $u_{2, j}$ can calculate $k_{2, j}$ without knowing any other's secret shares $\left\{k_{2, x}\right\}_{x \neq j}$ and satisfying that $k_{2, j}=K_{2}(j)$, where $K_{2}(j)$ is a random polynomial with constant $k g_{2}$ and degree $t_{2}-1$. $F_{2, j}(k)$ is a random polynomial privately owned by each $u_{2, j}$ with constant $s k t_{2, j}$ and degree $t_{3}-1$. $\left\{r_{2, j}=g^{k_{2, j}}\right.$ $\bmod p\} t_{2}$ and $r_{2}=g^{k_{2}} \bmod p$ are broadcast.

$\underline{u}_{3, k}$ runs $C T P$.

$$
\begin{gathered}
g^{s k t_{2, j, k}}=\left(\left(p k_{1} p k_{2, j}\right)^{e_{1}} r_{1} A\right)^{e_{2}} r_{2, j} B, \text { where } \\
B=\prod_{m=1}^{t_{3}-1}\left(g^{b_{2, j, m}}\right) k^{m} \bmod p \\
s k t_{2, k}^{\prime}=\sum_{j=1}^{t_{2}} \delta_{j} s k t_{2, j, k}=\sum_{j=1}^{t_{2}} \delta_{j} F_{2, j}(k) \\
=F_{2}^{\prime}(k), \text { where } \\
F_{2}^{\prime}(0)=\sum_{j=1}^{t_{2}} \delta_{j} F_{2, j}(0)=\sum_{j=1}^{t_{2}} \delta_{j} s k t_{2, j}=s k t g_{2} \\
\delta_{j}=\prod_{l=1, l \neq j}^{t_{2}}-l / j-l \\
s k p_{3, k}=e_{2} \cdot s k_{3, k}+s k t_{2, k}^{\prime} \bmod q .
\end{gathered}
$$

$\underline{U}_{3}$ runs $C T P S$.

$$
\begin{aligned}
& p s_{3, k}=c_{3} \cdot s k p_{3, k}+k_{3, k}, \text { where } \\
& c_{3}=H\left(1\|M\| p k g_{2}\left\|p k g_{3}\right\| \omega_{2} \| r_{1}, r_{2}\right), \text { and } \\
& r_{3}=g^{k_{3}} \bmod p . \\
& p \sigma_{3}=\sum_{u_{3, k} \in U_{3}} \delta_{k} \cdot p s_{3, k},
\end{aligned}
$$

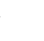




$$
\delta_{k}=\prod_{l=1, l \neq k}^{t_{3}}-l / k-l
$$

Verifier runs $C T P V$.

$$
\begin{aligned}
& g^{p \sigma_{3}}=P K P^{c_{3}} \cdot r_{3} \bmod p, \text { where } \\
& P K P=\left(p k_{1} \cdot p k g_{2}\right)^{e_{1}} \cdot r_{1}^{e_{2}} \cdot r_{2} \cdot p k g_{3}^{e_{2}} .
\end{aligned}
$$

PROOF OF (14) Let $\theta_{3}$ represents $u_{3, k} \in U_{3}$.

$$
\begin{aligned}
p \sigma_{3} & =\sum_{\theta_{3}} \delta_{k} p s_{3, k}=\sum_{\theta_{3}} \delta_{k}\left(c_{3}+s k p_{3, k}+k_{3, k}\right) \\
& =c_{3} \sum_{\theta_{3}} \delta_{k} s k p_{3, k}=\sum_{\theta_{3}} \delta_{k} k_{3, k} \\
& =c_{3} \sum_{\theta_{3}} \delta_{k}\left(e_{2} s k_{3, k}+s k t_{2, k}^{\prime}\right)+k_{3} \\
& =c_{3}\left(e_{2} \sum_{\theta_{3}} \delta_{3} s k_{3, k}+\sum_{\theta_{3}} \delta_{k} s k t_{2, k}^{\prime}\right)+k_{3} \\
= & c_{3}\left(e_{2} s k g_{3}+\sum_{\theta 2} \delta_{j} s k t_{2, j}\right)+k_{3} \\
= & c_{3}\left(e_{2} s k g_{3}+\sum_{\theta 2} \delta_{j}\left(e_{2} s k p_{2, j}+k_{2, j}\right)\right)+k_{3} \\
= & c_{3}\left(e_{2} s k g_{3}+e_{2} \sum_{\theta 2} \delta_{j} s k p_{2, j}+k_{2}\right)+k_{3} \\
= & c_{3}\left(e_{2} s k g_{3}+e_{2}\left(e_{1}\left(s k g_{2}+s k_{1}\right)+k_{1}\right)+k_{2}\right)+k_{3} \bmod q, \\
\text { L.H.S. } & =g^{p \sigma_{3}} \\
& =g^{c_{3}\left(e_{2} s k g_{3}+e_{2}\left(e_{1}\left(s k g_{2}+s k_{1}\right)+k_{1}\right)+k_{2}\right)+k_{3}} \bmod p \\
& =\text { R.H.S. }
\end{aligned}
$$

The proof proves the correctness of our CTPS scheme from the computation point of view. In the following section, we will prove its security.

\section{Security of the CTPS Scheme}

In the section, we set up CTPS security model and prove that our CTPS scheme is secure against adaptive chosen-message attack in random oracle model.

As discussed in [10], the formal model includes a rather powerful adversary who is able to corrupt all other users' secret keys except the Single Honest User (SHU). Furthermore, A is of the capability to launch adaptive chosen-message attacks according to three kinds of roles that the $S H U$ can play, namely Role 1: the original signer $u_{1}$, Role 2: one of the required proxy signers in $U_{2}$, say $u_{2,2}$, and Role 3: one of the required proxy signers in $U_{3}$, say $u_{3,2}$. All kinds of attacks will be described in the model later. Besides, A can access to a chained threshold proxy signing oracle. So the goal of the adversary A includes:

- A forgery CTPS on behalf of $u_{1}(S H U)$;

- A forgery CTPS by proxy signers in $U_{3}$, who are delegated by $U_{2}$ including $u_{2,2}(S H U)$, on behalf of $u_{1}$;

- A forgery CTPS by proxy signers including $u_{3,2}$ (SHU) in $U_{3}$ on behalf of $u_{1}$.

Definition 2 (Security of CTPS Scheme) Let CTPS = ( $G, K,(T D, T P),(C T D, C T P), C T P S, C T P V, C T P I D)$ be a chained threshold proxy signature scheme. Consider an experiment $\boldsymbol{E x p}_{C T P S, A}(k)$ related to CTPS, adversary $A$ and parameter $k$. In the extreme case, adversary $A$ should represent all proxy signers if the SHU is the original signer; or the original signer and all other proxy signers except the SHU if the SHU is one of the proxy signers in level 2 or 3. First, system parameters params and secret/public key pairs are generated by running $G$ and $K$. Empty array $\boldsymbol{s k p}_{3,2}$ and empty sets $\boldsymbol{p k g}_{2}$ and $\boldsymbol{p} \mathbf{k} \boldsymbol{g}_{3}$ are created. The adversary $A$ is of all the secret keys except SHU's, and it can make the following requests and queries.

R1: $u_{1}(S H U)$ designates $\left\{u_{2, j}\right\}_{n_{2}}$ and $\left\{u_{3, k}\right\}_{n_{3}}$ as his proxy signers. $A$ requests to interact with $u_{1}(S H U)$ running $T D$, and plays the role of $\left\{u_{2, j}\right\}_{n_{2}}$ running $T P$, and the roles of $U_{2}$ and $\left\{u_{3, k}\right\}_{n_{3}}$ running $(C T D, C T P)$. And $\boldsymbol{p k g}_{2}$ is set to $\boldsymbol{p} \boldsymbol{k} \boldsymbol{g}_{2} \cup p \mathbf{k g}_{2}$.

R2: $u_{1}$ designates $\left\{u_{2, j}\right\}_{n_{2}}$ and $\left\{u_{3, k}\right\}_{n_{3}}$, where $u_{2,2}$ is the $S H U$, as his proxy signers in level 2 and 3 respectively. $A$ requests to interact with $\left\{u_{2, j}\right\}_{n_{2}}$ running $T P$, and plays the role of $u_{1}$ running $T D$ and the roles of all other proxy signers in level 2 except $u_{2, j}$. Then $A$ requests again to interact with $u_{2,2}$ running $C T D$, and plays the role of $U_{2}$ except $u_{2,2}$, and $\left\{u_{3, k}\right\}_{n_{3}}$ running (CTD, CTP).

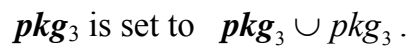

R3: $u_{1}$ designates $\left\{u_{2, j}\right\}_{n_{2}}$ and $\left\{u_{3, k}\right\}_{n_{3}}$, where $u_{3,2}$ is the $S H U$, as his proxy signers in level 2 and 3 respectively. $A$ requests to interact with $\left\{u_{3, k}\right\}_{n_{3}}$ running $C T P$, and plays the role of $U_{2}$ running $C T D$, and the roles of $u_{1}$ and $\left\{u_{2, j}\right\}_{n_{2}}$ running $(T D, T P)$. The private output $\boldsymbol{s k p}_{3,2}$ by $u_{3,2}(S H U)$ is stored in $\boldsymbol{s k p}_{3,2} . A$ does not have access to $\boldsymbol{s} \boldsymbol{k} \boldsymbol{p}_{3,2}$.

Q1: Chained threshold proxy signature query by $U_{3}$, where $u_{3,2}$ is the $S H U$, on behalf of $u_{1} . A$ can make a query $(M, 32, x)$ to oracle $O_{C T P S}\left(s k p_{3, k}\left(k=1,3, \cdots, t_{3}\right)\right.$, $\left.\boldsymbol{s k p}_{3,2}[x],,,,,\right)$. If $\boldsymbol{s k p}_{3,2}[x]$ has been defined, we say that this query is valid and the oracle returns $p \sigma_{3}=O_{C T P S}$ $\left(s k p_{3, k}\left(k=1,3, \cdots, t_{3}\right), \boldsymbol{s k p}_{3,2}[x], M, 32, x,\right)$. Eventually $A$ outputs a forgery $\left(M, p \sigma_{3}, p k_{1}\right)$. The output of the experiment is 1 , if

$$
\begin{aligned}
& -\operatorname{CTPID}\left(p \sigma_{3}\right) \backslash p \mathrm{pg}_{3} \notin \mathbf{p k g}_{2} \text {, or } \\
& \text { - CTPID }\left(p \sigma_{3}\right) \backslash p k g_{2} \notin \mathbf{p k g}_{3} \text {, or }
\end{aligned}
$$

- No valid query $(M, 32, x)$ to $O_{C T P S}\left(s k p_{3, k}(k=\right.$ $\left.\left.1,3, \cdots, t_{3}\right), \boldsymbol{s k p}_{3,2}[x], \because, \cdot,\right)$.

Otherwise, the output is 0 .

We define the advantage of adversary A as

$\boldsymbol{A d v}_{C T P S, A}(k)=\operatorname{Pr}\left[\boldsymbol{E x p}_{C T P S, A}(k)=1\right]$.

We say that CTPS is a secure chained threshold proxy signature scheme if the function $\boldsymbol{A} \boldsymbol{d} \boldsymbol{v}_{C T P S, A}(k)$ is negligible for $A$ of time complexity polynomial in the security parameter $k$.

SECURITY OF CTPS. The following theorem states 
our result about the security of Chained Threshold Proxy Signature scheme. The proof of Theorem 1 is in Appen$\operatorname{dix}$ A.

Theorem 1 Let CTPS $=(G, K,(T D, T P),(C T D, C T P)$, CTPS, CTPV, CTPID) be our proposed chained threshold proxy signature scheme in random oracle model. If the Schnorr signature scheme is secure, then CTPS scheme is secure in random oracle model.

PROOF IDEA. The conclusion that a Chained Threshold Proxy Signature scheme is provably secure can be deduced with respect to the contradiction that if a forgery of a chained threshold proxy signature scheme by $A$ successes in polynomial time, i.e. $\boldsymbol{A d v}_{C T P S, A}(k)$ is not negligible, then a well-known standard signature scheme, i.e. the Schnorr signature scheme, is broken.

\section{Chained Threshold Proxy Signature with Supervision (CTPSwS) Scheme}

Recall when Sam, the software development department, appoints Steven, the software maintenance department, as the supervising agent to supervise proxy signers such that only with permission of Steven, proxy signers can perform the signing capabilities. CTPSwS scheme in this section extended from CTPS fits into this kind of scenario by deploying Lui et al.'s [9] idea into our CTPS scheme.

Different from the CTPS model, there is a new protocol $\left(T D_{S A}, T P_{S A}\right)$ run between original signer $u_{1}$ and his supervising agent $S A_{1}$. To differentiate the protocol run between $u_{1}$ and $\left\{u_{2, j}\right\}_{n_{2}}$ in CTPSwS scheme with that in $C T P S$, we define the former as $\left(T D_{u}, T P_{u}\right)$. The signing and verification algorithm should also include $S A_{1}$ 's public key. The CTPSwS scheme model is as follows in Definition 3. Besides the notations described in Section 2, we have several new ones shown here.

$\left(s k_{S A_{1}}, p k_{S A_{1}}\right): S A_{1}$ 's secret and public key pair

$s k p_{S A_{1}}$ : partial proxy secret key generated by $S A_{1}$

$p s_{S A_{1}}$ : partial chained threshold proxy signature generated by $S A_{1}$

Definition 3 (CTPSwS Scheme) Let CTPSwS $=\{G$, $K, \quad\left(T D_{S A}, T P_{S A}\right), \quad\left(T D_{u}, T P_{u}\right), \quad(C T D, C T P), \quad C T P S w S$, $C T P V w S, C T P I D w S\}$ be a chained threshold proxy signature with supervision scheme, where the constituent algorithms run in polynomial time. $G$ and $K$ are similar to those in CTPS except that $K$ is also responsible to generate $S A_{1}$ 's secret/public key pair $\left(s k_{S A_{1}}, p k_{S A_{1}}\right)$.

$\left(T D_{S A}, T P_{S A}\right)$ is a Threshold Designation-Proxy protocol between the original signer $u_{1}$ and the supervising agent $S A_{1}$. Both $T D_{S A}$ and $T P_{S A}$ take as input the public keys $p k_{1}, p k g_{2}$ and $p k_{S A_{1}}$, respectively. $T D_{S A}$ also takes as input the secret key $s k_{1}$ of $u_{1}$, and $T P_{S A}$ takes as input the secret key $s k_{S A_{1}}$ of $S A_{1}$. As the result of the interaction, the expected local output of $T P_{S A}$ is $s k p_{S A_{1}}$, the partial proxy secret key of $S A_{1}$. The undeniability of both $u_{1}$ and $S A_{1}$ is achieved by adding $s k_{S A_{1}}$ in the protocol.

$$
\begin{gathered}
{\left[T D_{S A}\left(p k_{1}, p k g_{2}, p k_{S A_{1}}, s k_{1}\right),\right.} \\
\left.T P_{S A}\left(p k_{1}, p k g_{2}, p k_{S A_{1}}, s k_{S A_{1}}\right)\right] \rightarrow s k p_{S A_{1}} .
\end{gathered}
$$

$\left(T_{u}, T P_{u}\right)$ is a Threshold Designation-Proxy protocol between $u_{1}$ and $\left\{u_{2, j}\right\}_{n_{2}} . u_{1}$ runs $T D_{u}$ to send warrant $\omega_{1}$ to $\left\{u_{2, j}\right\}_{n_{2}} . T P_{u}$ run by each proxy signer $u_{2, j}$ takes as input the public keys $p k_{1}, p k g_{2}$ and the secret key $s k_{2, j}$ respectively. As the result of the interaction, the expected local output of $T P_{u}$ is $s k p_{2, j}$, the partial proxy secret share of $u_{2, j}$. Note the difference of this $s k p_{2, j}$ with that in CTPS scheme. We call it partial here because all $\left\{s k p_{2, j}\right\} t_{2}$ can not perform anything without another part $s k p_{S A_{1}}$.

$$
\left[T D_{w}, T P_{u}\left(p k_{1}, p k g_{2}, s k_{2, j}\right)\right] \rightarrow s k p_{2, j}
$$

$(C T D, C T P)$ is a Chained Threshold Designation-Proxy protocol between $U_{2}$ and $\left\{u_{3, k}\right\}_{n_{3}}$. It is similar to that is defined in Definition 1.

$$
\begin{gathered}
{\left[C T D\left(p k_{1}, p k g_{2}, p k_{S A_{1}}, p k g_{3},\left\{s k p_{2, j}\right\} t_{2}\right),\right.} \\
\left.C T P\left(p k_{1}, p k g_{2}, p k_{S A_{1}}, p k g_{3}, s k_{3, k}\right)\right] \rightarrow s k p_{3, k}
\end{gathered}
$$

CTPSwS is the (possibly) randomized Chained Threshold Proxy Signing with Supervision algorithm. It is run by $U_{3}$ with agreement of $S A_{1}$, and takes as input the $t_{3}$ out of $n_{3}$ corresponding partial proxy secret shares $\left\{s k p_{3, k}\right\} t_{3}$ and $S A_{1}$ 's partial proxy secret $s k p_{S A_{1}}$ and a message $M \in\{0,1\}^{*}$, and outputs a chained threshold proxy signature with supervision $p \sigma_{3}$.

$$
C T P S w S\left[\left\{s k p_{3, k}\right\} t_{3}, s k p_{S A_{1}}, M\right] \rightarrow p \sigma_{3}
$$

CTPVwS is the deterministic Chained Threshold Proxy Verification with Supervision algorithm as follows.

$$
C T P V w S\left[M, p \sigma_{3}, p k_{1}, p k g_{2}, p k_{S A_{1}}, p k g_{3}\right]=0 / 1
$$

CTPIDwS is the Chained Threshold Proxy IDentification with Supervision algorithm. It takes as input a valid proxy signature $p \sigma_{3}$, and outputs identities, i.e., public keys.

$$
\text { CTPIDwS }\left(p \sigma_{3}\right)=\left[\mathrm{pkg}_{2}, \mathrm{pk}_{\mathrm{SA}_{1}}, p k g_{3}\right] / \perp
$$

Based on Definition 3, we give a draft of a concrete $C T P S w S$ scheme here. Different from CTPS, $u_{1}$ needs to run $T D_{S A}$ to calculate $s k t_{1}$ and sends to his supervising agent $S A_{1}$. Different from Lui et al.'s idea, $S A_{1}$ runs $T P_{S A}$ to generate $s k p_{S A_{1}}=s k_{S A_{1}} e_{1}+s k t_{1} \bmod q$ using his secret key $s k_{S A_{1}}$. Without knowing $s k t_{1, j}$, each $u_{2, j}$ runs $T P_{u}$ to generate $s k p_{2, j}=s k_{2, j} e_{1} \bmod q .(C T D, C T P)$ run between 
$U_{2}$ and $\left\{u_{3, k}\right\}_{n_{3}}$ is the same as that in CTPS. At last when each $u_{3, k} \in U_{3}$ agrees to the data part, they must forward this email to $S A_{1}$, Steven, for him to check the contract part. $U_{2}$ can not generate a valid proxy signature on behalf of Sam until Steven agrees to the contract part and contributes his partial proxy signature $p s_{S A_{1}}=s k p_{S A_{1}} c_{3}+k_{S A_{1}} \bmod q$ where $k_{S A_{1}} \in{ }_{R} Z_{q}$ and $r_{S A_{1}}=g^{k_{S A_{1}}} \bmod q$. Since $S A_{1}$ 's secret key $s k_{S A_{1}}$ is involved, security level of our scheme is enhanced by adding $S A_{1}$-protected property.

\section{Security of CTPSwS}

The security model of CTPSwS is similar to that of CTPS defined in Definition 2, except that $A$ can be the fourth role, Role 4: the supervising agent of $u_{1}, S A_{1}$. In terms of this role, the goal of $A$ also includes a forgery CTPSwS by $U_{2}, U_{3}$ and $S A_{1}(S H U)$ on behalf of $u_{1}$.

Definition 4 (Security of CTPSwS) Let CTPSwS = $\left\{G, K,\left(T D_{S A}, T P_{S A}\right),\left(T D_{u}, T P_{u}\right),(C T D, C T P), C T P S w S\right.$, $C T P V w S, C T P I D w S\}$ be a chained threshold proxy signature scheme. Consider an experiment $\operatorname{Exp}_{C T P S w S, A}(k)$ related to CTPSwS, adversary $A$ and parameter $k$. In the extreme case, adversary A should represent all proxy signers and $S A_{1}$ if the $S H U$ is the original signer, or the original signer, $S A_{1}$, and all other proxy signers except the SHU if the SHU is one of the proxy signers in level 2 or 3, or the original signers and all proxy signers if the supervising agent $S A_{1}$ is the $S H U$. Empty arrays skp $\boldsymbol{s}_{3,2}$, $\boldsymbol{s k p}_{S A_{1}}$ and empty sets $\boldsymbol{p k g}_{2}, \boldsymbol{p k g}_{3}$ are created. The adversary A can make the following requests and queries:

R1: $u_{1}(S H U)$ designates $\left\{u_{2, j}\right\}_{n_{2}}$ and $\left\{u_{3, k}\right\}_{n_{3}}$ as his proxy signer groups in level $2 \& 3$, respectively, and specifies $S A_{1}$ as $u_{1}$ 's supervising agent. $A$ requests to interact with $u_{1}(S H U)$ running $T D_{S A}$ and $T P_{S A}$, and plays roles of all others running $\left(T D_{S A}, T P_{S A}\right)$ and $(C T D, C T P)$. $\boldsymbol{p k g}_{S A_{1}}$ is set to $\boldsymbol{p} \boldsymbol{k} \boldsymbol{g}_{S A_{1}} \cup p k g_{S A_{1}}$.

R2: $u_{1}$ designates $\left\{u_{2, j}\right\}_{n_{2}}$ and $\left\{u_{3, k}\right\}_{n_{3}}$ as his proxy signer groups in level $2 \& 3$, where $u_{2,2}$ is the $S H U$ and specifies $S A_{1}$ as $u_{1}$ 's supervising agent. $A$ requests to interact with $u_{2,2}(S H U)$ running $T P_{u}$ and $C T D$, and plays roles of all others running $\left(T D_{S A}, T P_{S A}\right), T D_{u}$ and $C T P$. $\boldsymbol{p k g}_{2}$ is set to $\boldsymbol{p} \boldsymbol{k g}_{2} \cup p \mathrm{~kg}_{2}$.

R3: $u_{1}$ designates $\left\{u_{2, j}\right\}_{n_{2}}$ and $\left\{u_{3, k}\right\}_{n_{3}}$ as his proxy signer groups in level $2 \& 3$, respectively, and specifies $S A_{1}(S H U)$ as $u_{1}$ 's supervising agent. $A$ requests to interact with $S A_{1}(S H U)$ running $T P_{S A}$, and plays roles of all others running the remanent algorithms and protocols. $\boldsymbol{s k} \boldsymbol{p}_{S A_{1}}$ is set to $\boldsymbol{s k} \boldsymbol{p}_{S A_{1}} \cup s k p_{S A_{1}}$.

R4: $u_{1}$ designates $\left\{u_{2, j}\right\}_{n_{2}}$ and $\left\{u_{3, k}\right\}_{n_{3}}$ as his proxy signer groups in level $2 \& 3$, where $u_{3,2}$ is the $S H U$, and specifies $S A_{1}$ as $u_{1}$ 's supervising agent. $A$ requests to interact with $u_{3,2}(S H U)$ running $C T P$, and plays roles of all others running the remanent algorithms and protocols. $\boldsymbol{s} \boldsymbol{k} \boldsymbol{p}_{3,2}$ is set to $\boldsymbol{s k} \boldsymbol{p}_{3,2} \cup s k p_{3,2}$.

Q1: Chained threshold proxy signature with supervision query, where $u_{3,2}$ is the $S H U$, on behalf of $u_{1}$. A can make a query $(M, 32, x)$ to oracle $O_{C T P W w S}\left(s k p_{3, k}(k\right.$ $\left.\left.=1,3, \cdots, t_{3}\right), \boldsymbol{s k p}_{3,2}[x], \operatorname{skp}_{S A_{1}}, \because, \cdot\right)$. If $\boldsymbol{s k p}_{3,2}[x]$ has been defined, we say this query is valid and the oracle returns $p \sigma_{3}=C T P S w \mathrm{~S}\left(s k p_{3, k}\left(k=1,3, \cdots, t_{3}\right), \boldsymbol{s k p}_{3,2}[x], \operatorname{skp} p_{S A_{1}}, M, \cdot, \cdot\right)$.

Q2: Chained threshold proxy signature with supervision query, where $S A_{1}$ is the $S H U$, on behalf of $u_{1}$. $A$ can make a query $\left(M, S A_{1}, x\right)$ to oracle $O_{C T P V w S}\left(s k p_{3, k}(k\right.$ $\left.\left.=1,2, \cdots, t_{3}\right), \boldsymbol{s k p}_{S A_{1}}[x], \because, ;\right)$. If $\boldsymbol{s k p}_{S A_{1}}[x]$ has been defined, we say this query is valid and the oracle returns $p \sigma_{3}=\operatorname{CTPSwS}\left(s k p_{3, k}\left(k=1,2, \cdots, t_{3}\right), \boldsymbol{s k p}_{S A_{1}}[x], M, \cdot, \cdot\right)$.

Eventually, $A$ outputs a forgery $\left(M, p \sigma_{3}, p k_{1}\right)$. The output of the experiment is determined as follows:

$$
\begin{aligned}
& \text { - CTPID } D_{w} S\left(p \sigma_{3}\right) \backslash\left(p k g_{3} \cup p k g_{S A_{1}}\right) \notin \boldsymbol{p k \boldsymbol { g } _ { 2 }} \text {, or } \\
& \text { - CTPID }{ }_{w} S\left(p \sigma_{3}\right) \backslash\left(p k g_{2} \cup p k g_{S A_{1}}\right) \notin \boldsymbol{p k g}_{3} \text {, or } \\
& \text { - No valid query }(M, 32, x) \text { to } O_{C T P V w S}\left(s k p_{3, k}(k\right. \\
& \left.\left.=1,3, \cdots, t_{3}\right), \mathbf{s k p}_{3,2}[x], \cdot, \cdot\right) \text {, } \\
& \text { - No valid query }\left(M, S A_{1}, x\right) \text { to } O_{C T P V W S}\left(\operatorname{skp}_{3, k}(k\right. \\
& \left.\left.=1,2, \cdots, t_{3}\right), \boldsymbol{s k p}_{S A_{1}}, \because, \cdot\right) \text {. }
\end{aligned}
$$

Otherwise, the output is 0 .

We define the advantage of adversary $A$ as

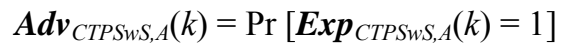

We say that $\boldsymbol{C T P S} \boldsymbol{w} \boldsymbol{S}$ is a secure chained threshold proxy signature with supervision scheme if the function

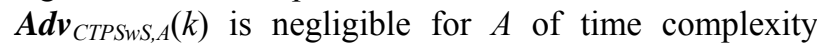
polynomial in the security parameter $k$.

Security proof details follow the similar logic as the CTPS scheme, but are more tedious and skipped in this paper.

\section{Conclusion and Discussion}

This paper designs two provably secure schemes in random oracle model, Chained Threshold Proxy Signature (CTPS) scheme and Chained Threshold Proxy Signature with Supervision (CTPSwS) scheme, to solve the group-to-group delegation problem and delegation with supervision in delegation chain problem. They are very useful in email with signature system where a manager wants to delegate his signing right to his vice managers, who can further perform the delegation to their employees. In some cases, the delegatee's proxy signing rights can be supervised by manager's supervising agent. For future work, we hope to develop more flexible delegation 
scheme that enables delegatees in different levels, say any one of vice managers and any two employees can cooperate to generate proxy signature. Also, more efficient schemes for these problems are always desirable.

\section{REFERENCES}

[1] Zoe L. Jiang, S. M. Yiu, L. C. K. Hui, Y. Dong, and S. H. Y. Wong, "Chained threshold proxy signature without and with Supervision," In 2008 International Conference on Computer Science and Software Engineering (CSSE’08), HongKong, China, pp. 837-840, December 2008.

[2] S. Kim, S. Park, and D. Won, "Proxy signatures, revisited," In 1st International Conference on Information and Communications Security (ICICS'97), LNCS 1334, Beijing, China, pp. 223-232, 1997.

[3] M. Mambo, K. Usuda, and E. Okamoto, "Proxy signatures for delegating signing operations," In 3rd ACM Conference on Computer and Communication Security, New Delhi, India, pp. 48-57, 1996.

[4] T. El Gamal, "A public key cryptosystem and a signature scheme based on discrete logarithms," In IEEE Transactions on Information Theory, Vol. 31, No. 4, pp. 469-472, 1985.

[5] T. Okamoto, "Provably secure and practical identification schemes and corresponding signature schemes," In Advances in Cryptology (Crypto'92), LNCS 740, pp. 31-53,
1992.

[6] A. Fiat and A. Shamir, "How to prove yourself: Practical solutions to identification and signature problems," In Advances in Cryptology-Eurocrypt 1986 (EuroCrypt'86), LNCS 263, pp. 186-194, 1987.

[7] B. C. Neuman, "Proxy-based authorization and accounting for distributed systems," In Proceedings of 13th International Conference on Distributed Computing Systems, Pittsburgh, USA, pp. 283-291, May 1993.

[8] M. Cerecedo, T. Matsumoto, and H. Imal, "Efficient and secure multiparty generation of digital signatures based on discrete logarithms," In IEICE Transactions on Fundamentals of Electronics, Communications \& Computer Science, Vol. E76-A, No. 4, pp. 532-545, 1993.

[9] R. W. C. Lui, L. C. K. Hui, and S. M. Yiu, "Delegation with supervision," In Information Sciences, Vol. 177, No. 19, pp. 4014-4030, 2007.

[10] A. Boldyreva, A. Palacio, and B. Warinschi, "Secure proxy signature schemes for delegation of signing rights," http://eprint.iacr.org/2003/096.

[11] A. Herzberg, S. Jarecki, H. Krawczyk, and M. Yung. "Proactive secret sharing or: How to cope with perpetual leakage," In Advances in Cryptology (Crypto'95), LNCS 963, Vol. 963, pp. 339-352, 1995.

[12] C. P. Schnorr, "Efficient signature generation by smartcards," In Journal of Cryptology, Vol. 4, No. 3, pp. 161174, 1991. 


\section{APPENDIX}

\section{A Proof of Theorem 1}

Suppose adversary $A$ is a successful forger against CTPS scheme in polynomial time. Let adversaries $B, C$, and $D$ wrap all communication channels from and to $A$, and have access to a standard signing oracle $O_{S}$, a chained threshold proxy signing oracle $O_{C T P S}$, and two random oracles functioning as hash functions $G$ and $H$ to answer $A$ 's requests and queries. First, system parameters params and secret/public key pairs are generated by running $G$ and $K$. Empty array $\boldsymbol{w s k p}_{3,2}$ and empty sets $\boldsymbol{p k g}_{2}$ and $\boldsymbol{p k g}_{3}$ are created. The adversary $A$ is of all the secret keys except $S H U$ 's, and it can make the following requests and queries.

R1: $A$ requests to interact with $u_{1}(S H U)$ running $T D$, and plays the role of $\left\{u_{2, j}\right\}_{n_{2}}$ running TP. The request is interrupted by $B$. $B$ creates an appropriate warrant $\omega_{1}$ and makes query $\left(0\left\|p k_{1}\right\| p k g_{2} \| \omega_{1}\right)$ to its signing oracle $O_{S}\left(s k_{1}, \cdot\right)$. Upon receiving an answer $\left(s k t_{1}, r_{1}\right)$, it forwards $\left(\omega_{1}, s k t_{1}, r_{1}\right)$ to $\left\{u_{2, j}\right\}_{n_{2}}$. After a successful run, $\boldsymbol{p k g}_{2}$ is set to $\boldsymbol{p k g}_{2} \cup \mathrm{pkg}_{2}$.

R2: $A$ requests to interact with $\left\{u_{2, j}\right\}_{n_{2}}$ running $T P$, where $u_{2,2}$ is the $S H U$, and plays the role of $u_{1}$ running TD. $C$ creates an appropriate warrant $\omega_{2}$ and makes query $\left(0\left\|p k g_{2}\right\| p k g_{3} \| \omega_{2}\right)$ to its signing oracle $O_{S}\left(s k p_{2,2},{ }^{\circ}\right)$. Upon receiving an answer $\left(s k t_{2,2}, r_{2}\right)$, it divides the answer into $n_{3}$ shares using random polynomial $F_{2,2}(x)$ and forwards $\left(\omega_{2},\left\{s k t_{2,2, k}\right\}_{n_{3}}, r_{2}\right)$ to $\left\{u_{3, k}\right\}_{n_{3}}$. $\boldsymbol{p k g}_{3}$ is set to $\boldsymbol{p k g}_{3} \cup \mathrm{pkg}_{3}$.

R3: $A$ requests to interact with $\left\{u_{3, k}\right\}_{n_{3}}$ running $C T P$, where $u_{3,2}$ is the $S H U$, and plays the role of $U_{2}$ running $C T D$. When $A$ outputs $\omega_{2}, s k t_{2, j, 2}, r_{2}, D$ verifies them. If all the verifications pass, $D$ stores $\left(\omega_{2}, s k t_{2,2}^{\prime}, r_{2}\right)$ in the last unoccupied position of $\boldsymbol{w s}_{\boldsymbol{k}} \boldsymbol{p}_{3,2}$.

Q1: $A$ can make a query $(M, 32, x)$ to oracle $O_{C T P S}\left(s k p_{3, k}\left(k=1,3, \cdots, t_{3}\right), \boldsymbol{s k} \boldsymbol{p}_{3,2}[x], ;, \cdot\right)$. If $\boldsymbol{w s k p}_{3,2}[x]$ is not defined, it returns $\perp$. Otherwise, $D$ performs the following operations:

- Pick a random number $c_{3} \in Z_{q}$.

- Pick a random number $p s_{3,2} \in Z_{q}$.

- Make a query (0\|pkg$\left.g_{2}\left\|p k g_{3}\right\| \omega_{2}, r_{2}\right)$ to oracle $G$ and let $e_{2}$ be the response.
- Compute commitment

$r_{3,2}=g^{p s_{3,2}}\left(p k_{3,2} e_{1} \cdot g^{s k t_{2, j}}\right)^{-c_{3}} \bmod p$

- Set $H\left(1\|M\| p k g_{2}\left\|p k g_{3}\right\| \omega_{2} \| r_{2}, r_{3}\right) \leftarrow \mathrm{c}_{3}$

- Return $\left(r_{3,2}, p s_{3,2}\right)$ to $A$

Suppose after the experiment $\operatorname{Exp}_{C T P S, A}(k), A$ outputs a forgery in polynomial time of $k$ such that at least one of the following events occurs:

E1. CTPID $\left(p \sigma_{3}\right) \backslash p k g_{3} \notin \boldsymbol{p k g}_{2}$.

E2. CTPID $\left(p \sigma_{3}\right) \backslash p k g_{2} \notin \boldsymbol{p k g}_{3}$.

E3. no valid query $(M, 32, x)$ to $O_{C T P S}\left(s k p_{3, k}(k=\right.$ $\left.\left.1,3, \cdots, t_{3}\right), \boldsymbol{s k p}_{3,2}[x], \cdot, \cdot\right)$.

Assume E1 occurs. Since all the coming in and out channels are wrapped by the adversaries, the query $H\left(1\|M\| p k g_{2}\left\|p k g_{3}\right\| \omega_{2} \| r_{2}, r_{3}\right)$ made by $A$ must be grabbed and responded by $\mathrm{c}_{3}$. By using forking lemma, $B$ can rewind $A$ to this point and gives $A$ another random $c_{3}^{\prime} \neq \mathrm{c}_{3}$. With non-negligible probability, $A$ produces a forgery with respect to the same query, such that

$$
\begin{aligned}
& g^{p \sigma_{3}}=P K P^{c_{3}} \cdot r_{3} \bmod p, \\
& g^{p \sigma_{3}^{\prime}}=P K P^{c_{3}^{\prime}} \cdot r_{3} \bmod p .
\end{aligned}
$$

Hence,

$$
\begin{aligned}
& g^{\left(p \sigma_{3}-p \sigma_{3}^{\prime}\right) \bmod q}=P K P^{\left(c_{3}-c_{3}^{\prime}\right) \bmod q} \bmod p, \\
& P K P=g^{S K P} \bmod p, \\
& S K P=e_{2} \cdot s k g_{3}+e_{2}\left(e_{1}\left(s k g_{2}+s k_{1}\right)+k_{1}\right)+k_{2} \bmod q .
\end{aligned}
$$

$B$ subtracts $e_{2} \cdot s k g_{3}+e_{2}\left(e_{1} \cdot s k g_{2}\right)+k_{2}$ for $B$ knows $s k g_{2}$ and $s k g_{3}$. Then it obtains $\sigma_{1}^{\prime}=s k_{1} \cdot e_{1}+k_{1} \bmod q$, a successful Schnorr signature of $u_{1}$.

Assume E2 occurs. The deduction is similar to the above. However since the $S H U$ is $u_{2,2}$, the adversary $C$ should subtract the corresponding parts, and obtain $\sigma_{2,2}^{\prime}$ $=s k_{2,2} \cdot e_{1}+k_{1} \bmod q$, a successful Schnorr signature of $u_{2,2}$.

Assume E3 occurs. The deduction is similar to the above. However since the $S H U$ is $u_{3,2}$, the adversary $D$ should subtract the corresponding parts, and obtain $\sigma_{3,2}^{\prime}$ $=s k_{3,2} \cdot e_{2}+k_{2} \bmod q$, a successful Schnorr signature of $u_{3,2}$. 